\title{
Oxytocin and the Gastrin-Releasing Peptide System in the Spinal Cord: Implications for Male Sexual Problems
}

\author{
Hirotaka SAKAMOTO* and Takumi OTI \\ Ushimado Marine Institute (UMI), Graduate School of Natural Science and Technology, Okayama University, \\ Ushimado, Setouchi, Okayama 701-4303, Japan
}

Received June 30, 2015; final version accepted July 22, 2015

\begin{abstract}
Neural circuits underlying male sexual function comprise several nuclei located in the brain and spinal cord. We have previously demonstrated in rats that the gastrin-releasing peptide (GRP) system influences spinal centers promoting penile reflexes. Moreover, a group of oxytocin (OXT) neurons, situated in the parvocellular part of the paraventricular nucleus of the hypothalamus, project into the spinal cord and control penile reflexes. Therefore, it has been hypothesized that OXT is transported by long descending paraventriculospinal pathways and activates proerectile spinal centers. Consequently, we have shown that in rats, axonal distribution of OXT in the lumbar spinal cord exhibits a male-dominant sexual dimorphism. Furthermore, OXT binding is observed in the spinal GRP neurons. Thus, OXT axons may secrete OXT from spinal axonal terminals and regulate male sexual function via an OXT receptor-mediated mechanism in spinal GRP neurons. Future studies should address the relationship between the hypothalamic OXT and spinal GRP systems. Identification of the male-specific brain-spinal cord neural circuit that regulates male sexual behavior may provide new avenues for therapeutic approaches to masculine reproductive dysfunction, including erectile dysfunction and/or ejaculation disorder.
\end{abstract}

KEYWORDS: oxytocin, gastrin-releasing peptide, spinal cord, male sexual behavior, neural circuit

\section{Introduction}

Neural circuits underlying male sexual function are composed of several nuclei located in the brain and spinal cord $[1,2]$. Lower spinal cord injuries frequently cause sexual dysfunction in men, including erectile dysfunction and ejaculation disorder $[3,4]$. This indicates that important neural centers for male sexual function are located within the lower spinal cord $[1,2]$. However, the molecular and neuroanatomical mechanisms controlling male sexual function at the lower spinal cord level remain unclear. We have previously identified a neuropeptide system expressing gastrinreleasing peptide (GRP) within the lumbosacral spinal cord that controls erection and ejaculation in male rats [5] (Fig. 1). This system of GRP neurons is sexually dimorphic, being prominent in male rats but vestigial or absent in females [5] (Fig. 1). Thus, it suggests that the sexually dimorphic GRP system in the lumbosacral spinal cord plays an important role in regulation of male sexual function [6].

A pertinent question is whether oxytocin (OXT) release triggers ejaculation in men as well as parturition and milk ejection in women. Hypothalamic OXT neurons projecting to the lower spinal cord may play an important role in control of penile reflexes and male sexual behavior in mammals [7]. Consequently, we are now examining the functional interaction between the newly identified spinal GRP system and hypothalamic OXT system. Identification of the male-specific brain-spinal cord neural circuit that regulates male sexual behavior may provide new avenues for therapeutic approaches to masculine reproductive dysfunction, including erectile dysfunction and/or ejaculation disorder [8].

In this review, we focus on the interaction between the spinal GRP system and hypothalamic OXT system as a model for the brain-spinal cord neural network controlling male sexual behavior.

\section{Identification of the GRP system and its local neural circuitry in the lumbosacral spinal cord}

During GRP immunohistochemical investigations, we identified a group of neurons in the lumbar spinal region (L3-L4 level) that play a role in erection and ejaculation [5, 9] (Fig. 2). These GRP-expressing neurons project axons to the more caudal spinal cord, and are more prominent in males than in females (Fig. 2).

Remarkably, pharmacological stimulation of GRP receptors (GRPRs) restores penile reflexes and ejaculation rate in castrated male rats, and antagonistic blockage to this spinal region attenuates penile reflexes and ejaculation rate in normal male rats [5]. To determine if androgen receptors (ARs) direct sexual dimorphism of these neurons, we next 


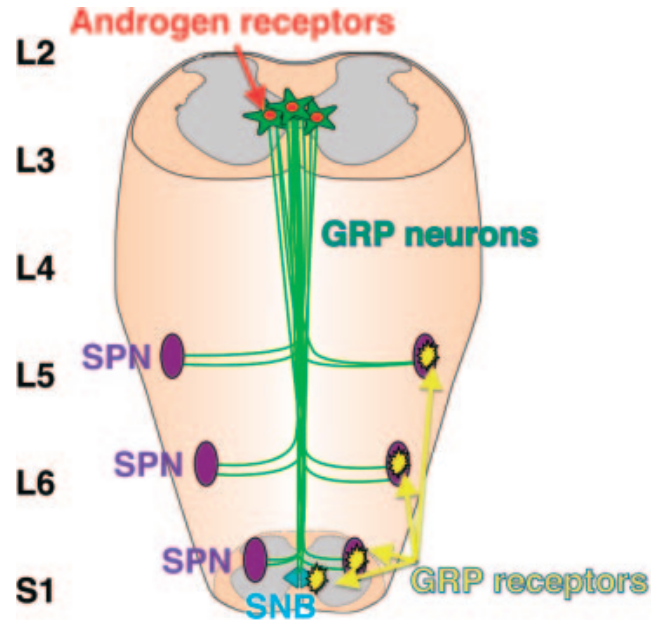

Fig. 1. Schematic drawing summarizing the gastrin-releasing peptide (GRP) system in the lumbosacral spinal cord that controls male sexual function. A sexually dimorphic spinal cord system of GRP-containing neurons in the upper lumbar spinal cord "ejaculation center" projects axons to both the autonomic (sacral parasympathetic nucleus; SPN) and somatic (spinal nucleus of the bulbocavernosus; SNB) centers in the lower lumbosacral spinal cord, which mediate penile reflexes and trigger ejaculation.

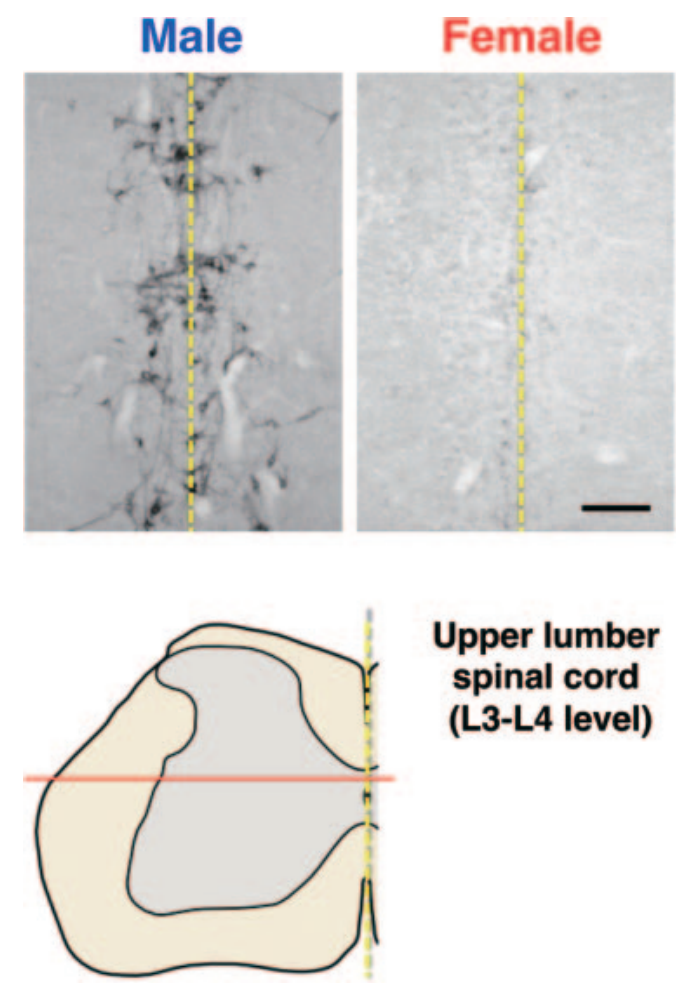

Fig. 2. GRP immunohistochemistry identifies the lumbosacral spinal cord system controlling male reproductive function. A collection of GRP-expressing neuronal cell bodies localized in the spinal cord lumbar segments 3 and 4 (L3-L4 level) project axons to the more caudal segments of the lumbosacral spinal cord (L5-S1 level), innervating the somatic and autonomic regions that are also known to control erection and ejaculation. Scale bar $=50 \mu \mathrm{m}$.

examined genetically male (XY) rats carrying the testicular feminization mutation (Tfm) of the Ar gene [10-12]. These rats embryologically develop testes and secrete testosterone prenatally; however, because they express dysfunctional AR protein, these rats develop a wholly feminine exterior phenotype, including a clitoris and defective vagina, rather than a penis and prostate $[10,11,13]$. The spinal cord of genetically male rats carrying the Tfm allele is hyperfeminine and exhibits even fewer GRP-positive neurons than dose the spinal cord of wild-type females. The spinal cord of Tfm males showed fewer GRP-positive neurons than that of wild-type males, as was expected [2,5]. In addition, GRPexpressing neurons in males express ARs but do not express estrogen receptors [5]. Thus, this neuronal system in the lumbosacral spinal cord is androgen-dependent and specifically uses the GRP peptide to drive lower spinal autonomic and somatic centers that coordinate male reproductive functions such as erection and ejaculation $[1,6,14]$. 
In male rats, the spinal nucleus of the bulbocavernosus (SNB) is a cluster of motor neurons located in the fifth and sixth lumbar and first sacral segments of the spinal cord (L5-S1 level) [2,15-17]. These motor neurons and their target muscles, the bulbocavernosus and levator ani, which are attached to the base of the penis, play an important role in male copulatory behavior [15,17-19]. It has been demonstrated that adult male rat castration leads to the somatic and dendritic shrinkage of SNB motoneurons and reduction in the number of synaptic inputs, all of which can be prevented by treating castrates with exogenous testosterone [20-24]. Moreover, treatment of ovariectomized female rats with exogenous testosterone administered in adulthood increases cellular sizes of SNB motoneurons, although not to the level seen in males $[17,20]$. These results are consistent with those of the spinal GRP system in terms of low-sensitivity for androgens in adult females [5, 17, 25,26], and consequently suggest that both testosterone and AR, as an androgen signaling system, are necessary to maintain both the GRP and SNB systems in the lumbosacral spinal cord [2,6, 17, 25]. Additionally, these two systems at the same lumbosacral spinal level may directly interact because the sexually dimorphic distribution of GRP-positive fibers in the lower lumbar and upper sacral spinal cord (L5-S1 level) is profoundly regulated by circulating androgens [26], and mirrors the changes in SNB motoneuron arborization and synapse number $[21,22,27]$. We three-dimensionally examined at the ultrastructural level, the axodendritic synaptic inputs of spinal GRP neurons to SNB motoneurons, using high-voltage electron microscopy (HVEM) [28]. HVEM is a promising approach for studying chemical neuroanatomy at the ultrastructural level, and its results can be linked to light microscopic observations [29,30]. The method involves pre-embedding immunoelectron microscopy combined with a retrograde tracing technique injecting a cholera toxin $\beta$ subunit-horseradish peroxidase conjugate from the bulbocavernosus muscles in order to visualize SNB motoneurons. By tilting a thick epoxy-resin-embedded section (1$5 \mu \mathrm{m}$ order), HVEM tomography is a powerful method for resolution of three-dimensional ultrastructure [29,30]. By tilting the specimen stage $\pm 8^{\circ}$, HVEM analysis in rats provided a clear three-dimensional visualization of synaptic contacts from the GRP system in the lumbosacral spinal cord to the dendrites of SNB motoneurons [28, 30] (Fig. 3). Contraction of the bulbocavernosus muscles is involved in both penile erection and ejaculation in rats [31], supporting the idea that GRP-containing afferents to SNB motoneurons may control penile reflexes during sexual behavior through the identified synapses. Accordingly, activation and functional synchronization of both spinal systems are required for normal penile reflexes [14]. Using HVEM tomography, we also found that the terminals of GRP neurons can form multiple axonal projections with the dendrites of sacral parasympathetic nucleus (SPN) neurons [32] (Fig. 4).

Moreover, using a viral trans-synaptic retrograde tracing technique, Dobberfuhl et al. [33] recently reported that after pseudorabies virus (PRV) injection into the levator ani muscle, approximately half of PRV-positive neurons in the medial gray at the L3-L4 level expressed GRP (Fig. 5). Taken together, these results indicate that the local neural circuit in the lumbosacral spinal cord, which includes GRP-expressing neurons, plays a pivotal role in the regulation of male sexual function. In addition, it has been reported that GRPRs are expressed in both the SPN and SNB [5,34]. Thus, we recently generated a transgenic rat expressing red fluorescent protein under the control of GRPR promoter.

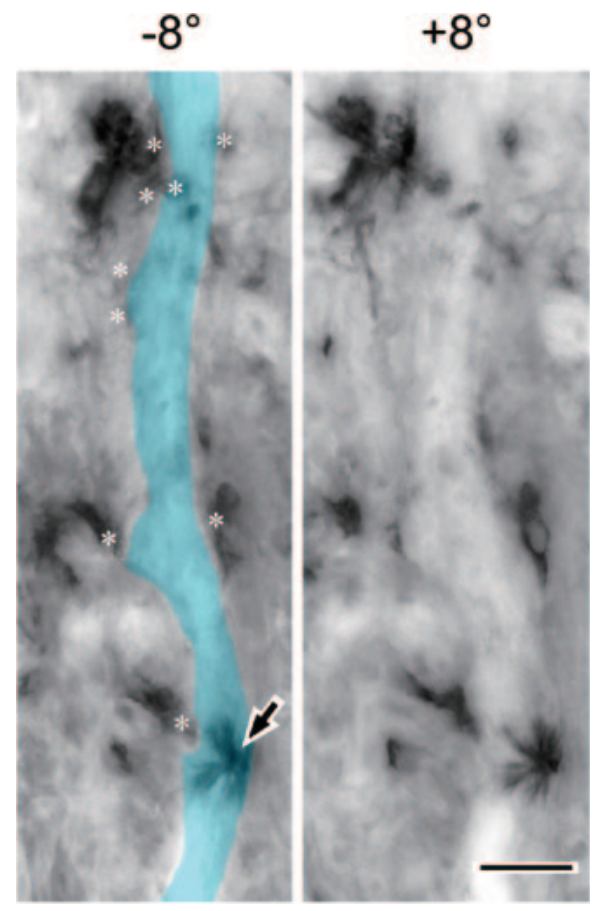

Fig. 3. Stereo-paired high-voltage electron microscopy (HVEM) images obtained by tilting the specimen stage $\pm 8^{\circ}$ identify threedimensional GRP axonal projections (white asterisks) into a single SNB dendrite. The arrow points to an electron-dense tetramethylbenzidine reaction product in a SNB dendrite displaying the characteristic, radial structure. Scale bar $=2 \mu \mathrm{m}$. 


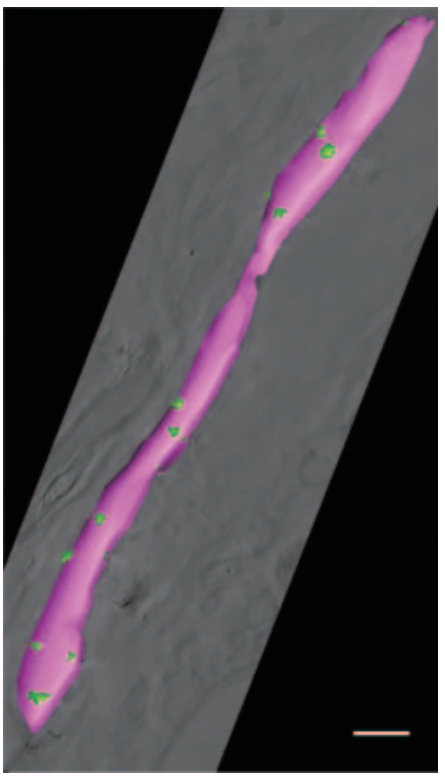

Fig. 4. Three-dimensional reconstruction of HVEM images using a dual immunoelectron microscopic technique for GRP (green) and a SPN neuronal dendrite (magenta). Scale bar $=2 \mu \mathrm{m}$.
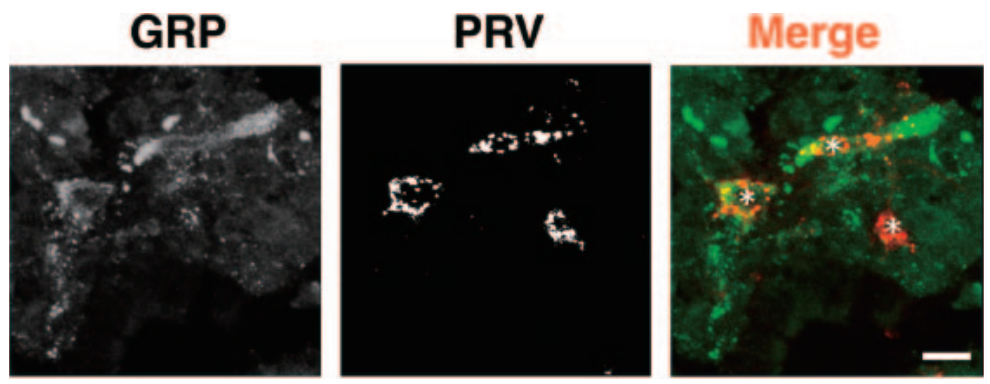

Fig. 5. Double immunofluorescence for GRP and pseudorabies virus (PRV) in the lumbar spinal cord (L3-L4 level) after the injection of PRV into the levator ani muscle. GRP-immunoreactivity and PRV-immunoreactivity are merged in the right panel. Asterisks $(*)$ indicate the location of neuronal nuclei expressing GRP and/or PRV. Scale bar $=20 \mu \mathrm{m}$.

Preliminary results show higher fluorescent signals in SNB motoneurons in the lumbosacral spinal cord of transgenic rats, with the signals showing a male dominant sexual dimorphism (our unpublished observations). Therefore, the spinal GRP/GRPR system could generate an ejaculatory response by activating autonomic and somatic centers e.g., SPN and SNB, in the lumbosacral spinal cord [34]. These findings support the hypothesis that the GRP/GRPR system may regulate the male sexual behavior via afferents to both SPN and SNB neurons and organize autonomic and somatic functions, in response to penile reflexes during male sexual behavior [34].

\section{Hypothalamic OXT system controls male sexual function}

OXT is mainly produced by magnocellular neurosecretory cells in the paraventricular nucleus (PVN) and supraoptic nucleus of the hypothalamus and released into the circulation from axon terminals distributed in the posterior pituitary [35]. The primary functions of OXT are the regulation of female reproductive functions including parturition, milk ejection, and maternal behavior; therefore, OXT has been implicated mainly in the physiological functions in females (for review, see Ref. 35).

However, a group of OXT neurons originating from the PVN and projecting to extra-hypothalamic areas might be accepted to control penile erection and ejaculation in male rats [7,36-39]. OXT has also been reported to positively impact upon a number of components of sexual function in men, including libido, erection, and orgasm [40-42]. Intracerebroventricular administration of OXT induces a dose-dependent increase in the number of penile erections and yawning episodes in male rats [43]. Because penile erection and yawning induced by either OXT or apomorphine were antagonized by pretreatment with OXT antagonists in a dose-dependent manner, dopamine may induce these responses by releasing OXT [43]. Furthermore, OXT concentration in rat cerebrospinal fluid doubles $5 \mathrm{~min}$ after ejaculation, and increases to three times the basal level 20 min after ejaculation [44]. In men, mean plasma OXT also increases at the 
time of ejaculation, but not during sexual arousal $[45,46]$. This OXT increase in cerebrospinal fluid at ejaculation is eliminated in rats by discrete electrolytic lesions in the lateral and posterior parvocellular PVN [44]. Importantly, with increasing levels of sexual contact, c-fos expression is enhanced in OXT neurons in the parvocellular hypothalamic PVN [47]. Parvocellular PVN neuron-specific chemical lesions in rats significantly reduce the density of OXTcontaining fibers in the lower lumbar and upper sacral spinal cord (L5-S1 level), and result in attenuated ejaculation function [48]. Therefore, it has long been postulated that OXT, which is transported by long descending paraventriculospinal pathways, activates proerectile spinal centers [49]; however, direct linkage of the neural circuit between the hypothalamic PVN and penile innervation remains uncharacterized [39].

Notably, we found that axonal distribution and local OXT concentration in the lumbar spinal cord exhibits a maledominant sexual dimorphism in rats (Fig. 6). In contrast, no sexual difference was detected for vasopressin whose expression is relatively low in the lumbar spinal cord. Furthermore, OXT binding was observed in the cell bodies of spinal GRP neurons (Fig. 7).

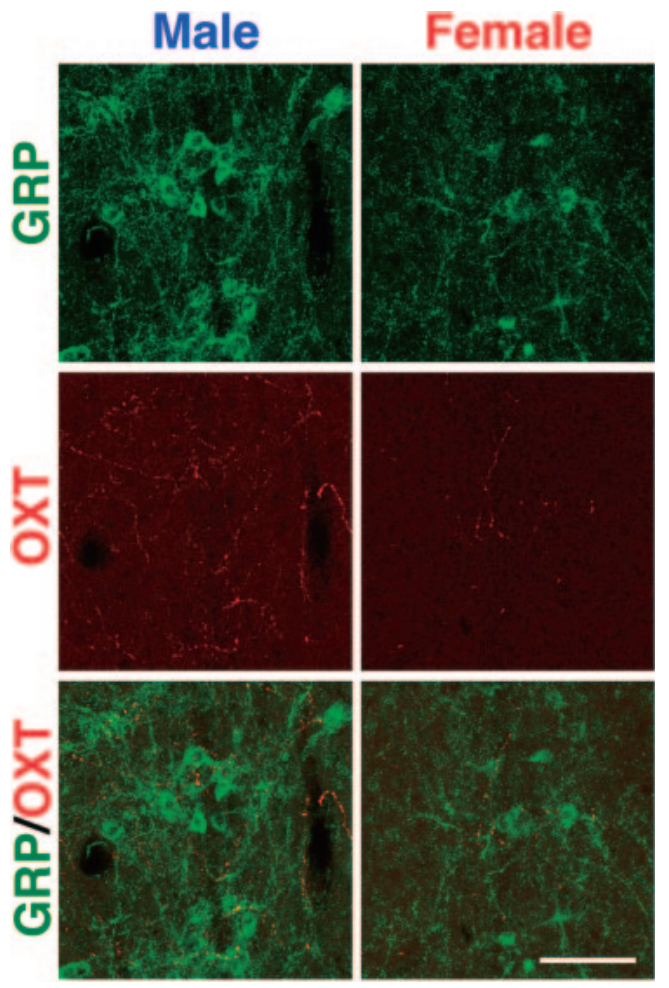

Fig. 6. Double immunofluorescence for GRP (green) and oxytocin (OXT) (red) shows that OXT-containing axons surrounding spinal GRP neurons are prominent in male rats but vestigial or absent in females. Scale bar $=100 \mu \mathrm{m}$.

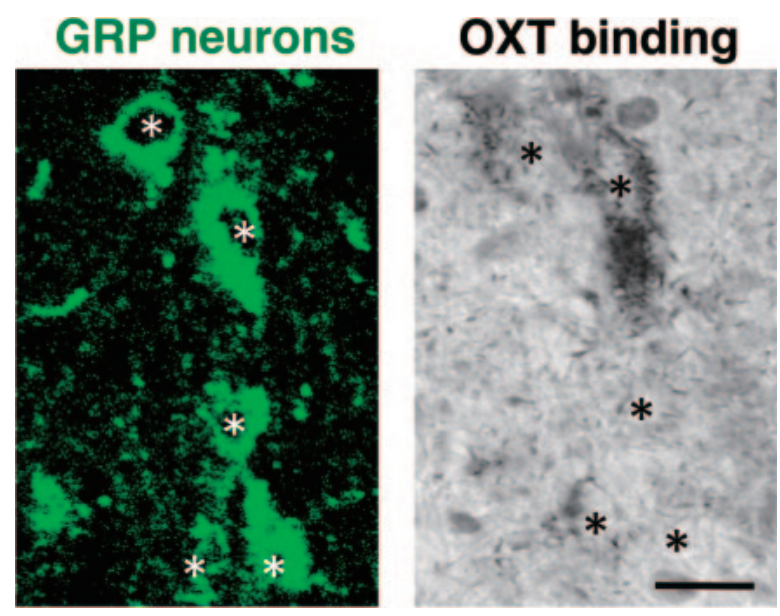

Fig. 7. OXT binding is particularly apparent in spinal GRP neurons (immunolabeled in green). Asterisks (*) indicate the location of neuronal nuclei expressing GRP and OXT binding. Scale bar $=20 \mu \mathrm{m}$. 
Consequently, we suggest that OXT axons may secrete OXT from terminals distributed in the lumbar spinal cord and that male sexual function may be regulated through an OXT receptor-mediated mechanism in the spinal GRP neurons [39]. Future studies should focus on the relationship between the hypothalamic OXT and spinal GRP systems to identify the brain-spinal cord neural network controlling male sexual function. Interestingly, there may be minimal functional synaptic contacts between OXT axons and GRP neurons in the lumbar spinal cord: most OXT neurons in the PVN remained unlabeled by retrograde trans-synaptic tracing with PRV from the bulbocavernosus muscle in experiments performed to identify neural circuits controlling male sexual function [50,51]. This suggests that OXTcontaining axons in the lumbar spinal cord may function via a non-synaptic mechanism, i.e., the volume transmission.

\section{Concluding remarks}

This paper reviews our recent efforts to examine the brain-spinal cord neural circuitry controlling male sexual behavior. Here, we propose a novel hypothesis that parvocellular OXT axons of the PVN secrete OXT from terminals distributed in the lumbosacral spinal cord, and that male sexual reflexes, such as erection and ejaculation, are regulated through an OXT receptor-mediated mechanism in the spinal GRP neurons during copulatory behavior (see Fig. 8). Future studies should focus on the relationship between the hypothalamic OXT and spinal GRP systems. Improved understanding of the brain-spinal cord neural interaction controlling male sexual function may open the door to a new field of research for behavioral neuroendocrinology and clinical investigations.

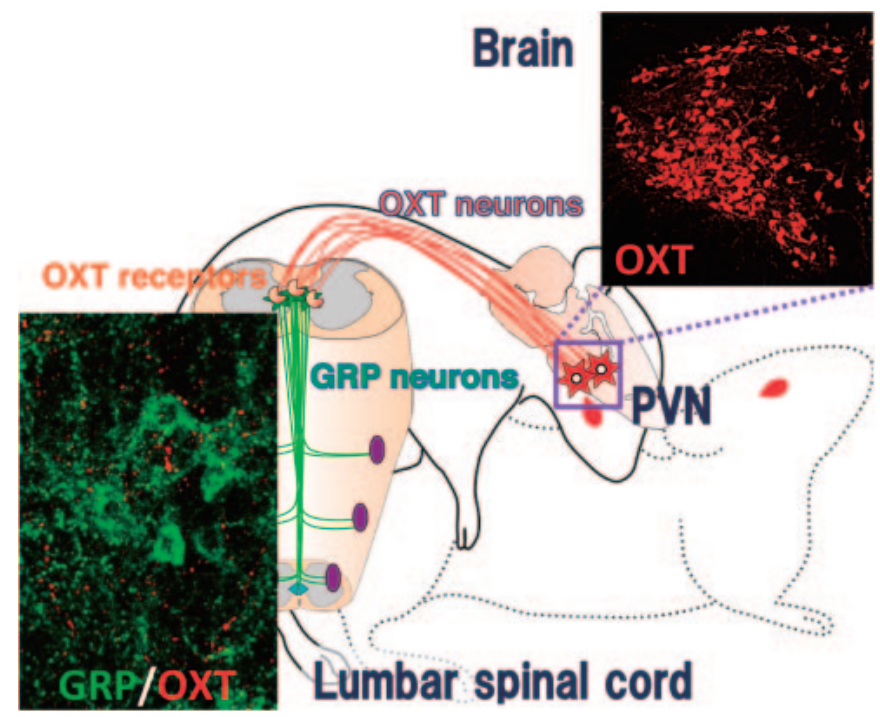

Fig. 8. A novel hypothesis in terms of the OXT-GRP system controlling male sexual function in the lumbosacral spinal cord. During male sexual behavior, hypothalamic OXT axons may trigger ejaculation via an OXT receptor-mediated mechanism in the spinal GRP system.

\section{Acknowledgments}

This work was supported in part by JSPS/KAKENHI from the Ministry of Education, Science, Sports, Culture and Technology (MEXT), Japan to H.S.; by the Naito Memorial Grant for Natural Science Researches, Japan to H.S.; by the Kato Memorial Bioscience Foundation, Japan to H.S.; by the Life Science Foundation of Japan to H.S.; and by the Co-operative Study by High-voltage Electron Microscopy (H-1250M) of the National Institute for Physiological Sciences, Okazaki, Japan to H.S. and T.O. T.O. is supported by a Research Fellowship of JSPS for Young Scientists. The authors would like to thank Enago (www.enago.jp) for the English language review. Disclosure of Summary: The authors have nothing to disclose.

\section{REFERENCES}

[1] Sakamoto H (2012) Brain-spinal cord neural circuits controlling male sexual function and behavior. Neurosci Res 72:103-116.

[2] Sakamoto H (2014) Sexually dimorphic nuclei in the spinal cord control male sexual functions. Front Neurosci 8:184 eCollection 2014.

[3] Sipski ML (1998) Sexual functioning in the spinal cord injured. Int J Impot Res 10 Suppl 2:S128-130; discussion S138-140.

[4] Brown DJ, Hill ST, Baker HW (2006) Male fertility and sexual function after spinal cord injury. Prog Brain Res 152:427-439.

[5] Sakamoto H, Matsuda K-I, Zuloaga DG, Hongu H, Wada E, Wada K, Jordan CL, Breedlove SM, Kawata M (2008) Sexually dimorphic gastrin releasing peptide system in the spinal cord controls male reproductive functions. Nat Neurosci 11:634-636. 
[6] Sakamoto H, Kawata M (2009) Gastrin-releasing peptide system in the spinal cord controls male sexual behaviour. J Neuroendocrinol 21:432-435.

[7] Argiolas A, Melis MR (2005) Central control of penile erection: role of the paraventricular nucleus of the hypothalamus. Prog Neurobiol 76:1-21.

[8] Sakamoto H (2010) The neurobiology of psychogenic erectile dysfunction in the spinal cord. J Androl 31:519-526.

[9] Truitt WA, Coolen LM (2002) Identification of a potential ejaculation generator in the spinal cord. Science 297:1566-1569.

[10] Bardin CW, Bullock L, Blackburn WR, Sherins RJ, Vanha-Perttula T (1971) Testosterone metabolism in the androgeninsensitive rat: a model for testicular feminization. Birth Defects Orig Artic Ser 7:185-192.

[11] Yarbrough WG, Quarmby VE, Simental JA, Joseph DR, Sar M, Lubahn DB, Olsen KL, French FS, Wilson EM (1990) A single base mutation in the androgen receptor gene causes androgen insensitivity in the testicular feminized rat. J Biol Chem 265:8893-8900

[12] Quigley CA, De Bellis A, Marschke KB, el-Awady MK, Wilson EM, French FS (1995) Androgen receptor defects: historical, clinical, and molecular perspectives. Endocr Rev 16:271-321.

[13] Zuloaga DG, Puts DA, Jordan CL, Breedlove SM (2008) The role of androgen receptors in the masculinization of brain and behavior: what we've learned from the testicular feminization mutation. Horm Behav 53:613-626.

[14] Sakamoto H (2011) The gastrin-releasing peptide system in the spinal cord mediates masculine sexual function. Anat Sci Int 86:19-29.

[15] Breedlove SM, Arnold AP (1980) Hormone accumulation in a sexually dimorphic motor nucleus of the rat spinal cord. Science 210:564-566.

[16] Forger NG, Breedlove SM (1986) Sexual dimorphism in human and canine spinal cord: role of early androgen. Proc Natl Acad Sci U S A 83:7527-7531.

[17] Sengelaub DR, Forger NG (2008) The spinal nucleus of the bulbocavernosus: firsts in androgen-dependent neural sex differences. Horm Behav 53:596-612.

[18] Breedlove SM, Arnold AP (1983) Hormonal control of a developing neuromuscular system. I. Complete Demasculinization of the male rat spinal nucleus of the bulbocavernosus using the anti-androgen flutamide. J Neurosci 3:417-423.

[19] Breedlove SM, Arnold AP (1983) Hormonal control of a developing neuromuscular system. II. Sensitive periods for the androgen-induced masculinization of the rat spinal nucleus of the bulbocavernosus. J Neurosci 3:424-432.

[20] Breedlove SM, Arnold AP (1981) Sexually dimorphic motor nucleus in the rat lumbar spinal cord: response to adult hormone manipulation, absence in androgen-insensitive rats. Brain Res 225:297-307.

[21] Kurz EM, Sengelaub DR, Arnold AP (1986) Androgens regulate the dendritic length of mammalian motoneurons in adulthood. Science 232:395-398.

[22] Matsumoto A, Micevych PE, Arnold AP (1988) Androgen regulates synaptic input to motoneurons of the adult rat spinal cord. J Neurosci 8:4168-4176.

[23] Goldstein LA, Kurz EM, Sengelaub DR (1990) Androgen regulation of dendritic growth and retraction in the development of a sexually dimorphic spinal nucleus. J Neurosci 10:935-946.

[24] Yang LY, Verhovshek T, Sengelaub DR (2004) Brain-derived neurotrophic factor and androgen interact in the maintenance of dendritic morphology in a sexually dimorphic rat spinal nucleus. Endocrinology 145:161-168.

[25] Forger NG (2009) The organizational hypothesis and final common pathways: Sexual differentiation of the spinal cord and peripheral nervous system. Horm Behav 55:605-610.

[26] Sakamoto H, Takanami K, Zuloaga DG, Matsuda KI, Jordan CL, Breedlove SM, Kawata M (2009) Androgen regulates the sexually dimorphic gastrin-releasing peptide system in the lumbar spinal cord that mediates male sexual function. Endocrinology 150:3672-3679.

[27] Matsumoto A (2001) Androgen stimulates neuronal plasticity in the perineal motoneurons of aged male rats. J Comp Neurol 430:389-395.

[28] Sakamoto H, Arii T, Kawata M (2010) High-voltage electron microscopy reveals direct synaptic inputs from a spinal gastrinreleasing peptide system to neurons of the spinal nucleus of bulbocavernosus. Endocrinology 151:417-421.

[29] Hama K, Kosaka T (1981) Neurobiological applications of high voltage electron microscopy. Trends Neurosci 4:193-196.

[30] Sakamoto H, Kawata M (2012) Ultrahigh voltage electron microscopy links neuroanatomy and neuroscience/neuroendocrinology. Anat Res Int 2012:Article ID: 948704.

[31] Sachs BD (1982) Role of striated penile muscles in penile reflexes, copulation, and induction of pregnancy in the rat. J Reprod Fertil 66:433-443.

[32] Oti T, Satoh K, Saito K, Murata K, Kawata M, Sakamoto T, Sakamoto H (2012) Three-dimensional evaluation of the spinal local neural network revealed by the high-voltage electron microscopy: a double immunohistochemical study. Histochem Cell Biol 138:693-697.

[33] Dobberfuhl AD, Oti T, Sakamoto H, Marson L (2014) Identification of CNS neurons innervating the levator ani and ventral bulbospongiosus muscles in male rats. J Sex Med 13:664-677.

[34] Takanami K, Sakamoto H (2014) The gastrin-releasing peptide receptor (GRPR) in the spinal cord as a novel pharmacological target. Curr Neuropharmacol 12:434-443.

[35] Russell JA, Leng G (1998) Sex, parturition and motherhood without oxytocin? J Endocrinol 157:343-359.

[36] Wagner CK, Clemens LG (1991) Projections of the paraventricular nucleus of the hypothalamus to the sexually dimorphic lumbosacral region of the spinal cord. Brain Res 539:254-262.

[37] Wagner CK, Clemens LG (1993) Neurophysin-containing pathway from the paraventricular nucleus of the hypothalamus to a sexually dimorphic motor nucleus in lumbar spinal cord. J Comp Neurol 336:106-116.

[38] Tang Y, Rampin O, Calas A, Facchinetti P, Giuliano F (1998) Oxytocinergic and serotonergic innervation of identified lumbosacral nuclei controlling penile erection in the male rat. Neuroscience 82:241-254. 
[39] Sakamoto H, Satoh K, Oti T (2012) Achieve orgasm? Oxytocin triggers ejaculation in men. Reprod Sys Sexual Disorders $1: 1000 \mathrm{e} 1001$.

[40] Burri A, Heinrichs M, Schedlowski M, Kruger TH (2008) The acute effects of intranasal oxytocin administration on endocrine and sexual function in males. Psychoneuroendocrinology 33:591-600.

[41] Ishak WW, Berman DS, Peters A (2008) Male anorgasmia treated with oxytocin. J Sex Med 5:1022-1024.

[42] MacDonald K, Feifel D (2012) Dramatic improvement in sexual function induced by intranasal oxytocin. J Sex Med 9:1407-1410.

[43] Melis MR, Argiolas A, Gessa GL (1989) Evidence that apomorphine induces penile erection and yawning by releasing oxytocin in the central nervous system. Eur J Pharmacol 164:565-570.

[44] Hughes AM, Everitt BJ, Lightman SL, Todd K (1987) Oxytocin in the central nervous system and sexual behaviour in male rats. Brain Res 414:133-137.

[45] Carmichael MS, Humbert R, Dixen J, Palmisano G, Greenleaf W, Davidson JM (1987) Plasma oxytocin increases in the human sexual response. J Clin Endocrinol Metab 64:27-31.

[46] Murphy MR, Seckl JR, Burton S, Checkley SA, Lightman SL (1987) Changes in oxytocin and vasopressin secretion during sexual activity in men. J Clin Endocrinol Metab 65:738-741.

[47] Witt DM, Insel TR (1994) Increased Fos expression in oxytocin neurons following masculine sexual behavior. J Neuroendocrinol 6:13-18.

[48] Ackerman AE, Lange GM, Clemens LG (1997) Effects of paraventricular lesions on sex behavior and seminal emission in male rats. Physiol Behav 63:49-53.

[49] Rousselot P, Papadopoulos G, Merighi A, Poulain DA, Theodosis DT (1990) Oxytocinergic innervation of the rat spinal cord. An electron microscopic study. Brain Res 529:178-184.

[50] Tang Y, Rampin O, Giuliano F, Ugolini G (1999) Spinal and brain circuits to motoneurons of the bulbospongiosus muscle: retrograde transneuronal tracing with rabies virus. J Comp Neurol 414:167-192.

[51] Veronneau-Longueville F, Rampin O, Freund-Mercier MJ, Tang Y, Calas A, Marson L, McKenna KE, Stoeckel ME, Benoit G, Giuliano F (1999) Oxytocinergic innervation of autonomic nuclei controlling penile erection in the rat. Neuroscience 93:1437-1447. 